\title{
The role of primary healthcare physicians in the COVID-19 pandemic: a cross-sectional study
}

\author{
Rami Saadeh $^{1}$, Mahmoud Alfaqih ${ }^{1}$, Amjad Al Shdaifat ${ }^{2}$, Mohammad Alyahya ${ }^{3}$, Nasr \\ Alrabadi $^{3}$, Yousef Khader ${ }^{3}$, Othman Beni Yonis ${ }^{3}$, and Mohammed Allouh ${ }^{4}$ \\ ${ }^{1}$ Jordan University of Science and Technology \\ ${ }^{2}$ The Hashemite University \\ ${ }^{3}$ Jordan University of Science and Technology Faculty of Medicine \\ ${ }^{4}$ United Arab Emirates University
}

November 3, 2020

\begin{abstract}
Background: Following the remarkable spread of coronavirus disease 2019 (COVID-19), worldwide, it quickly became apparent that many public health systems worldwide were not prepared to manage such a pandemic. We aimed to assess the perceptions of primary care physicians (PCPs) in Jordan toward their role during COVID-19. Methods: A cross-sectional study using a self-administered questionnaire was performed. The study participants included PCPs from the Ministry of Health, academic institutions, and the private sector in Jordan. Results: A total of 221 PCPs participated in the study. Most participants reported not having received any training on infection control (59.7\%) or COVID-19 (81\%). More than half PCPs (53.4\%) felt positive about the way patients received and/or complied with their instructions. More than half PCPs (55.7\%) educated their patients on protective measures against COVID-19 infection and considered this as part of their role and responsibility. Over $80 \%$ of the participants would apply social distancing, hand sanitation, facial masks, and patient education, but only half $(51.1 \%)$ reported planning to order COVID-19 test kits. Conclusions: PCPs had a positive attitude toward controlling COVID-19 infection and showed a willingness to educate patients on how to protect themselves. However, PCPs should be provided special training on COVID-19.
\end{abstract}

\section{Hosted file}

Role of PCPs in COVID-19.pdf available at https://authorea.com/users/372461/articles/490489the-role-of-primary-healthcare-physicians-in-the-covid-19-pandemic-a-cross-sectionalstudy 